\title{
$\mathrm{Pt} / \mathrm{TiO}_{2}$ 上苯和乙烯光催化氧化过程的磁场效应
}

\author{
杨俊伟王绪绪戴文新李旦振付贤智* \\ (福州大学光催化研究所, 福州 350002)
}

\begin{abstract}
摘要 以 $\mathrm{Pt} / \mathrm{TiO}_{2}$ 为催化剂和 $365 \mathrm{~nm}$ 紫外光为光源, 在低于 $0.2 \mathrm{~T}$ 的磁场强度范围考察了磁场对苯和乙烯光催 化氧化反应的影响. 实验发现, 当用紫外灯管作光源并靠近磁场磁极或者置于磁场磁极间隙内照射反应器时, 磁场可显著提高气相苯和乙烯的光催化转化率和矿化率; 而当光源远离磁场磁极而通过光导纤维传导照射到 反应器上时, 磁场对这两个反应没有促进作用. 在前一种情况下, 紫外灯管的发光强度随磁场的增强而提高, 磁 场对这两个光催化降解反应的促进作用被证实是由于磁场提高了紫外灯管的发光强度, 进而为反应提供了更 多的光能和使反应温度升高所致; 在后一种光照方式下, 由于光源不受磁场影响, 磁场对这两个光催化反应不 产生影响. 这一研究结果表明, 在所研究磁场强度范围, 磁场对气-固相光催化反应没有任何本征影响.
\end{abstract}

关键词：光催化，磁场效应，苯，乙烯

中图分类号：0643

\section{Effect of Magnetic Field on the Photocatalytic Oxidation of Benzene and Ethylene over Pt/TiO}

\author{
YANG, Jun-Wei $\quad$ WANG, Xu-Xu $\quad$ DAI, Wen-Xin $\quad$ LI, Dan-Zhen $\quad$ FU, Xian-Zhi* \\ (Research Institute of Photocatalysis, Fuzhou University, Fuzhou 350002, P. R. China)
}

\begin{abstract}
The effect of magnetic field on the photocatalytic oxidation of benzene and ethylene contained in air over $\mathrm{Pt} / \mathrm{TiO}_{2}$ was studied under $365 \mathrm{~nm}$ irradiation in the range of magnetic field intensity of $\leqslant 0.2 \mathrm{~T}$. It was found that when the UV lamp was near to or under a magnetic field, the magnetic field could enhance the photocatalytic conversion and mineralization of benzene and ethylene. However, no effect of magnetic field on the reactions was observed when the UV lamp was far away from the magnetic field and the light was transmitted onto the reactor by a focusing fiber. In the former case, the observed enhance of photocatalytic oxidation of the two reactants was a result of the increase of brightness of the UV lamp under magnetic field. This is because that high photo intensity provided high light energy for the reaction and led to increase of the reaction temperature. In the latter case, intensity of UV light wasn't disturbed by the magnetic field, applying magnetic field had no effect on the two photocatalytic reactions. The results showed that there was no any inherent effect on the gas-solid phase photocatalytic reaction.
\end{abstract}

Keywords: Photocatalysis, Magnetic field effect, Benzene, Ethylene

量子效率低是光催化技术难以大规模应用的最 大障碍. 为解决这一问题, 国内外研究者进行了大量 探索. 除了设计和开发新型催化剂和对 $\mathrm{TiO}_{2}$ 半导体 进行体相及表面组成和结构修饰外, 不少研究还表 明, 给光催化反应体系附加其它外场(电场、微波场、
超声波场等)也可以提高光催化过程的效率 ${ }^{[1-3]}$. 作者 曾报道 ${ }^{\left[{ }^{1}\right.}$, 磁场也能提高苯气相光催化降解反应的 效率, 发现用 $\mathrm{Pt} / \mathrm{TiO}_{2}$ 作催化剂, 在 $60 \mathrm{mT}$ 的磁场和 $254 \mathrm{~nm}$ 紫外光的共同作用下, 苯的氧化转化率可由 15.5\%提高到 $18.0 \%$, 矿化率由 $19.0 \%$ 提高到 $52.0 \%$.

Received: June 13, 2005; Revised :June 21, 2005. *Correspondent, E-mail : xzfu@fzu.edu.cn; Tel:0591-83738608.

国家自然科学基金(20133010), 国家重大基础研究前期研究专项(2004CCA07100), 福建省重大基础研究(No.2003F004)资助项目

C. Editorial office of Acta Physico-Chimica Sinica 
随后, Wakasa 等 ${ }^{[5]}$ 研究了磁场对 $\mathrm{TiO}_{2}$ 悬浮体系中叔 丁醇光催化降解反应的影响, 发现施加 $1.5 \mathrm{~T}$ 的磁 场能使叔丁醇的氧化转化率提高大约 $10 \%$. 然而, 关于磁场影响光催化过程的原因都没能给出确切的 解释. 我们 ${ }^{[4]}$ 曾经推断, 磁场对苯气相光催化过程的 影响可能是由于磁场通过影响自由基的电子排布状 态, 改变了反应的途径和加速了中间产物的转化. Wakasa 等 ${ }^{[5}$ 通过比较有无磁场下反应体系吸收光谱 变化认为, 磁场不是通过影响自由基而是通过阻碍 光生电子和空穴的复合提高了光催化过程的效率. 基于一般光催化过程的自由基机理特征, 并综合以 前的这些研究以及有关光化学反应磁场效应的研究 结果, 我们推测磁场对光催化过程的影响远比想象 的要复杂. 最近我们在实验中发现, 当磁场与紫外光 同时施加到反应体系且彼此靠得很近时, 磁场会显 著影响紫外光源的发光光强, 这在以前的相关研究 中都没有提及和考察过. 因此, 要确定磁场效应及认 识其本质尚需要深人细致的实验观察和分析.

本文设计了两种磁场-光催化反应装置, 以 $\mathrm{Pt} /$ $\mathrm{TiO}_{2}$ 为催化剂在 $365 \mathrm{~nm}$ 的紫外光照射下, 分别考 察了磁场对苯和乙烯光催化氧化转化率和矿化情况 的影响. 但这两种装置上得到结果却完全不同, 效果 取决于反应装置中磁场磁极和光源的布局方式. 详 细的观察和比较发现, 磁场通过影响光源的发光强 度影响光催化反应; 当排除磁场对光源的影响时, 磁 场对这些有机物的光催化氧化反应并不产生影响.

\section{1 实验部分}

\section{1 催化剂制备}

参考文献[6]是通过改进的溶胶-凝胶法制备 $\mathrm{TiO}_{2}$ 干凝胶. 催化剂 $\mathrm{Pt} / \mathrm{TiO}_{2}$ 样品通过对干凝胶进 行如下处理和修饰制备: 研磨并篎分出粒径 50 70 目 (直径 $0.2 \sim 0.3 \mathrm{~mm}$ ) 的颗粒, 按照 $n(\mathrm{Pt}): n\left(\mathrm{TiO}_{2}\right)=$ 0.005:1 的比例用 $\mathrm{H}_{2} \mathrm{PtCl}_{6}$ 溶液进行浸渍处理, 烘干 和焙烧后再用摩尔浓度为 $0.1 \mathrm{~mol} \cdot \mathrm{L}^{-1}$ 的 $\mathrm{NaBH}_{4}$ $\mathrm{NaOH}$ 混合溶液进行还原处理 ${ }^{[7]}$, 最后用去离子水洗 涤至 $\mathrm{pH}$ 为 7 8, $110{ }^{\circ} \mathrm{C}$ 烘干备用.

\section{2 光催化实验装置和条件}

图 1 为实验所用两种磁场-光催化反应装置的 示意图, 分别用 $(M)$ 和 $(N)$ 表示. 在装置(M)中, 石英反 应管置于反应器中部, 内装催化剂, 外围均匀置有四 根主波长为 $365 \mathrm{~nm}$ 的紫外灯; 一个自制的电磁铁 的磁极置于灯管的上下间隙(磁场强度通过电压调
节, $220 \mathrm{~V}$ 电压下最大磁场强度为 $60 \mathrm{mT}$; 反应管内 催化剂床层的长度小于磁场磁极直径, 磁场方向垂 直于反应管放置方向); 整个反应装置被置于一个圆 柱套管内. 在反应装置 $(\mathrm{N})$ 中, 催化剂装在一个扁平 矩形状(厚 $0.3 \mathrm{~cm} \times$ 长 $3 \mathrm{~cm} \times$ 宽 $1.5 \mathrm{~cm}$ ) 石英反应器 中, 置于磁场两磁极的中央; 光源(SHG-200, Mejiro precision Inc., 秋灯, 波长 $365 \mathrm{~nm}$ ) 由液心光纤和聚 焦头输出, 输出光为 $1.5 \mathrm{~cm} \times 1.5 \mathrm{~cm}$ 的矩形光斑, 可 完全覆盖反应器, 聚焦头距反应器表面约 $7 \mathrm{~cm}$, 强 度为 $15.89 \mathrm{~mW} \cdot \mathrm{cm}^{-2}$; 磁场也为一个电磁铁(吉林长 春英普公司), 但场强可通过调节磁极间距在 $0 \sim 1.5$ $\mathrm{T}$ 范围改变, 由于反应器的宽度限制, 装置最大只能 给反应器施加 $0.2 \mathrm{~T}$ 的磁场. 两个装置中光催化反 应器的温度均通过插人催化剂床层的热电偶测量. 两装置的主要差别为: 在反应装置 $(\mathrm{M})$ 中, 磁极与紫 外灯管之间的距离很近; 而在反应装置 $(\mathrm{N})$ 中, 光源 远离磁场并采用光纤引人.

反应物苯和乙烯分别由钢瓶提供, 用干净空气 配置. 含苯空气中的苯浓度为 $665 \mu \mathrm{L} \cdot \mathrm{L}^{-1}$, 含乙烯空 气中的乙烯浓度为 $640 \mu \mathrm{L} \cdot \mathrm{L}^{-1}$. 反应物气体先后通 过稳压阀和稳流阀以恒定的流速 $\left(20 \mathrm{~mL} \cdot \mathrm{min}^{-1}\right)$ 连续 流人反应器, 出口处的苯浓度和 $\mathrm{CO}_{2}$ 含量采用气相 色谱(HP6890, Porapak R) 在线测定. 在线测定结果 是重复的. 反应体系所加磁场的磁场强度由数字磁 强计(LX-H-2 型, 北京宏拓控制技术有限责任公司) 测定, 测试时其探头紧贴反应管催化剂床层外壁. 催 化剂的受光强度用紫外辐照计(Nim Sun UV Meter 型, 中国计量科学研究院) 测量. 光的波长在外部用 FL/FS900 时间分辨苂光光谱仪测量.

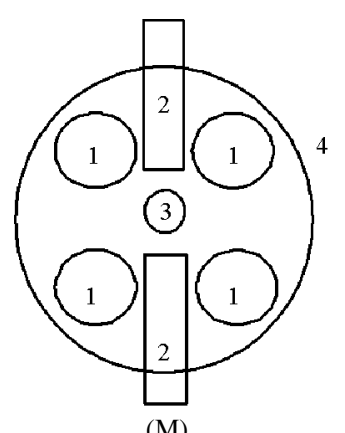

图 1 磁场-光催化反应装置

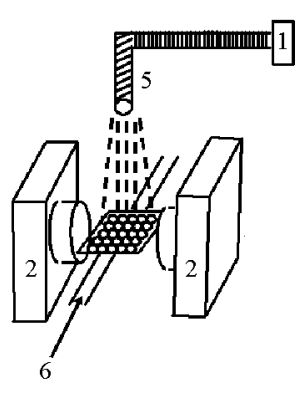

(N)
Fig.1 Apparatus for magnetic-photocatalytic reaction

1) UV lamp; 2) magnetic pole; 3) quartz reactor; 4) bushing; 5) light fiber; 6) reaction gas 

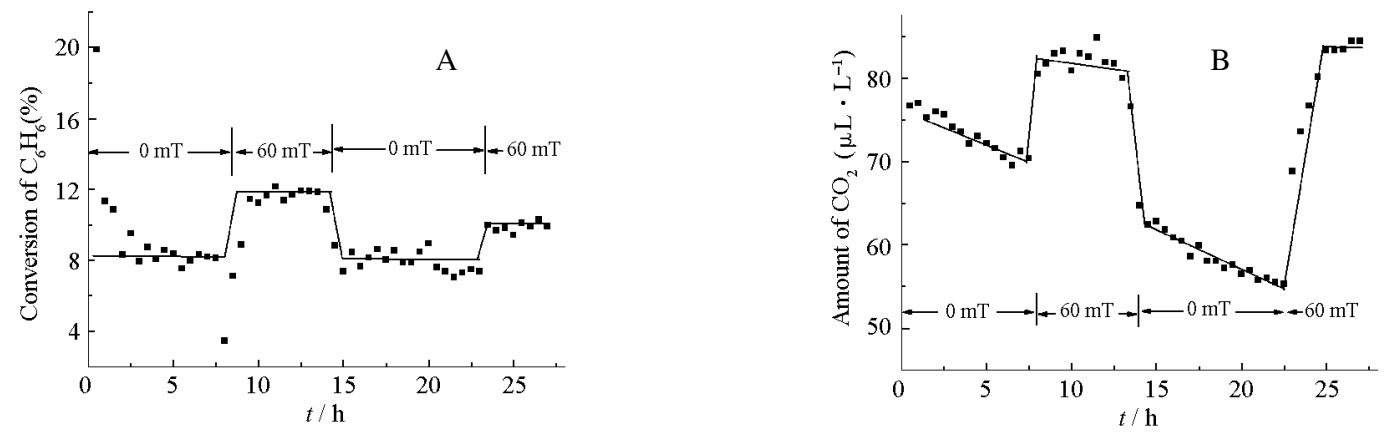

图 2 用反应装置(M)所观测外加磁场对苯光催化转化率和 $\mathrm{CO}_{2}$ 产量的影响

Fig.2 Effect of added magnetic field on the photocatalytic conversion of benzene and $\mathrm{CO}_{2}$ yield on $\mathrm{Pt} / \mathrm{TiO}_{2}$ with the apparatus (M)

A) benzene conversion; B) $\mathrm{CO}_{2}$ yield

\section{2 结果与讨论}

\section{1 不同磁场-光催化反应装置上苯和乙烯气相光 催化氧化效果比较}

首先用反应装置 $(M)$ 考察了磁场对 $\mathrm{Pt} / \mathrm{TiO}_{2}$ 光催 化降解苯的影响. 苯的转化率和氧化产物 $\mathrm{CO}_{2}$ 产量 在加磁场和不加磁场时的变化见图 2. 可以看出, 在 主波长为 $365 \mathrm{~nm}$ 的紫外光作用下, 反应基本达稳 态时的苯转化率为 $7.70 \%, \mathrm{CO}_{2}$ 的产量为 $57.13 \mu \mathrm{L}$. $\mathrm{L}^{-1}$. 随后当施加 $60 \mathrm{mT}$ 的磁场并达到新的稳态时, 苯的转化率会提高到 $11.69 \%, \mathrm{CO}_{2}$ 的浓度也随之增 加到 $83.20 \mu \mathrm{L} \cdot \mathrm{L}^{-1}$. 然后关闭磁场时, 苯的稳态转化 率便恢复到以前的水平. 随后再次施加 $60 \mathrm{mT}$ 磁场, 苯的转化率又随之有较小幅度增加. 开始, 即未加磁 场阶段苯转化率的下降以及苯转化率提高幅度在第 二次施加磁场时比第一次施加磁场时的小, 可能与 催化剂随反应时间的延长而逐渐失活有关. $\mathrm{CO}_{2}$ 的 浓度增加在第二次施加磁场时比第一次施加磁场高 的原因将在后面讨论, 可能与表面沉积物的增多和 反应温度的升高有关. 这与我们以前在 $254 \mathrm{~nm}$ 光照
下所观察到磁场对苯光催化反应的影响相吻合 ${ }^{[4]}$.

施加磁场对相同条件下乙烯光催化氧化反应也 有显著的正影响(图 3). 当单纯光催化过程达稳态后 也施加 $60 \mathrm{mT}$ 磁场, 乙烯的稳态转化率由 $29.58 \%$ 上 升至 $68.07 \%$. 随后关闭磁场, 乙烯转化率基本恢复 到先前的水平. 接着依次施加 $38 \mathrm{mT}$ 磁场, 达到稳 态后再提高磁场强度到 $48 \mathrm{mT}$, 乙烯转化率出现阶 梯式提高. 对乙烯的氧化, 催化剂的失活较小, 反应 在每个阶段都可达到稳态. $\mathrm{CO}_{2}$ 的产量也随施加磁 场而提高, 从消耗乙烯的量和 $\mathrm{CO}_{2}$ 的产量计算, 乙 烯被完全矿化.

如果去掉装置(M)中的铁磁铁, 将灯管和反应 管部分固定在装置 $(\mathrm{N})$ 所用电磁铁的磁极间隙内, 在 $60 \mathrm{mT}$ 磁场强度下可观察到苯和乙烯的光催化转化 率有类似的变化趋势, 变化值更加明显. 当试图用此 装置进一步考察更强磁场对反应的影响时, 却发现 紫外灯管的亮度随磁场强度的提高而增加, 且当磁 场达到一定强度和紫外灯管达到一定亮度时再增大 磁场, 灯管开始闪㷧随后亮度逐渐减弱以致最后熄
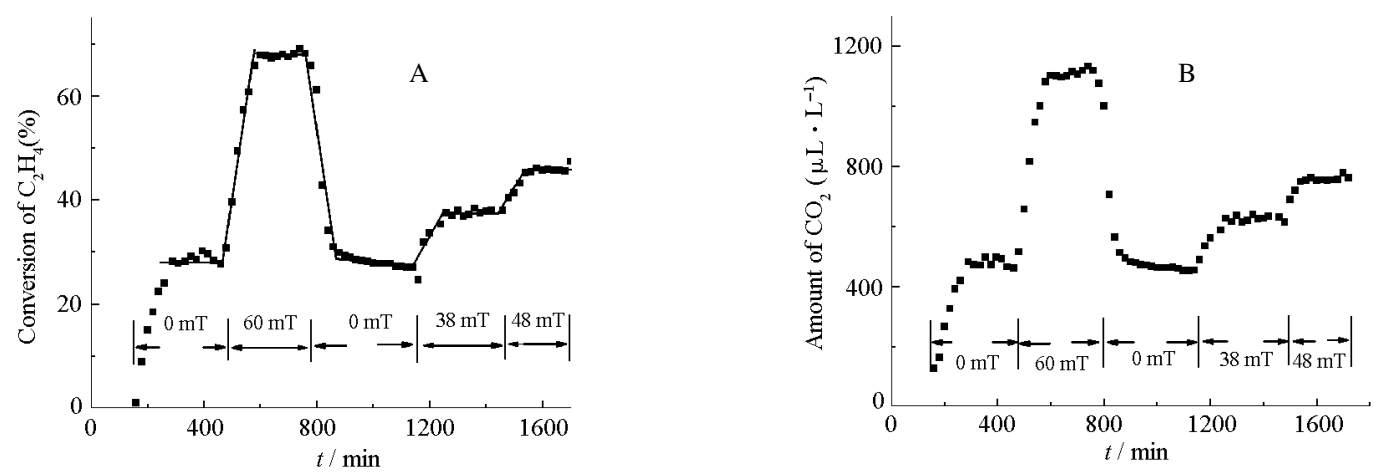

图 3 用反应装置 $(\mathbf{M})$ 所观测磁场对乙烯光催化转化率和 $\mathrm{CO}_{2}$ 产量的影响

Fig.3 Effect of added magnetic field on the photocatalytic conversion of ethylene and $\mathrm{CO}_{2}$ yield on $\mathrm{Pt} / \mathrm{TiO}_{2}$ with the apparatus (M)

A) ethylene conversion, $\mathrm{B}$ ) $\mathrm{CO}_{2}$ yield 

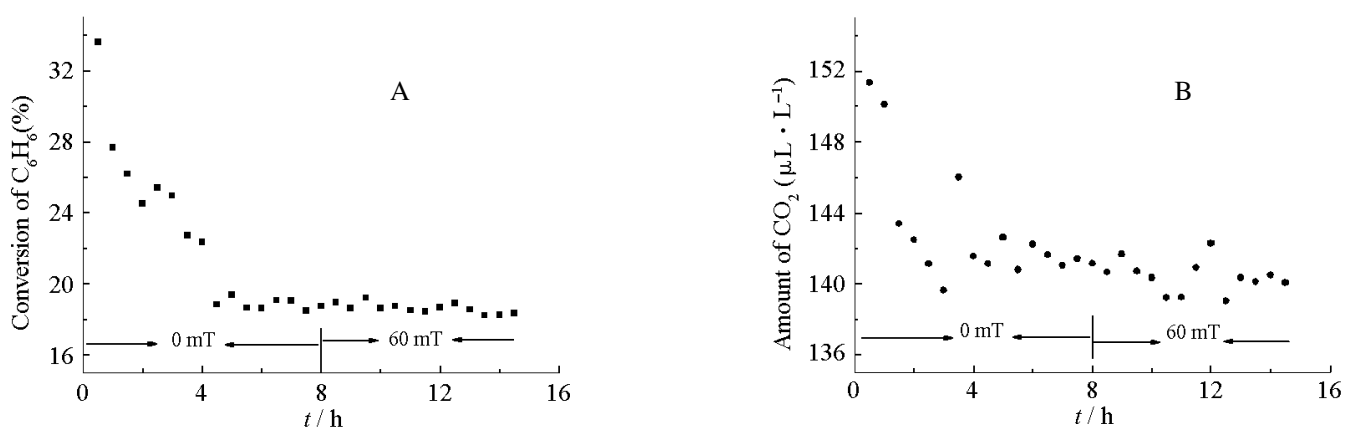

图 4 用反应装置(N)所观察磁场对苯光催化转化率和 $\mathrm{CO}_{2}$ 产量的影响

Fig.4 Effect of magnetic field on the photocatalytic conversion of benzene and $\mathrm{CO}_{2}$ yield over $\mathrm{Pt} / \mathrm{TiO}_{2}$ with the apparatus $(\mathrm{N})$

A) benzene conversion; $\mathrm{B}$ ) $\mathrm{CO}_{2}$ yield

灭. 这使我们怀疑上面所观察到光催化磁场效应可 能与磁场对光源的影响有关. 众所周知, 光催化过程 的活化能来源于光能, 光强的增强对反应有利. 那 么, 要揭示磁场对光催化过程的本征影响, 应该排除 其对光强的影响, 于是设计了反应装置 $(\mathrm{N})$ 并用其再 一次考察了磁场对苯和乙烯光催化反应的影响. 图 4 和图 5 分别是用反应装置 $(\mathrm{N})$ 所测定磁场对苯和 乙烯在 $365 \mathrm{~nm}$ 光照下和 $\mathrm{Pt} / \mathrm{TiO}_{2}$ 上的光催化降解转 化率和 $\mathrm{CO}_{2}$ 产量变化. 可以看出, 当光催化反应达 稳态时施加 $60 \mathrm{mT}$ 磁场, 苯和乙烯的转化率并不出 现图 2 和图 3 中所表现出的突跃, 施加磁场前后几 乎看不出有明显的差别.

上面的实验结果表明, 排除磁场对光源的影响 后, 施加 $60 \mathrm{mT}$ 磁场不会对光催化效应起到促进作 用. 那么施加更强的磁场是否会对气相光催化过程 产生影响呢?

\section{2 磁场强度对苯和乙烯气相光催化氧化的影响}

实验部分已经提到, 当矩形反应管放置磁极间 时, 把磁极间距调到最小时只能最大获得 $0.2 \mathrm{~T}$ 的

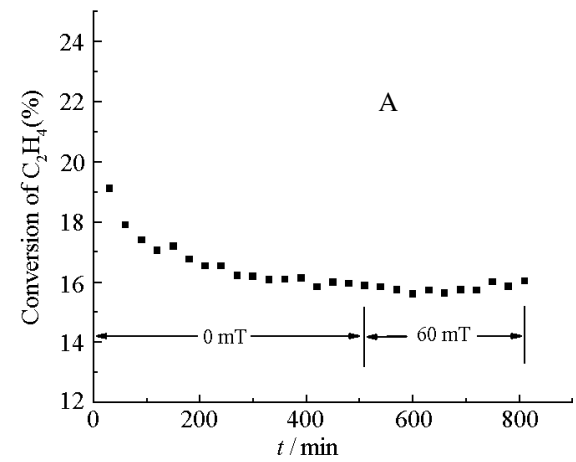

磁场, 但这已经比上述实验所用磁场的强度高 3 倍 多. 然而, 在强度 $0.2 \mathrm{~T}$ 磁场作用下, 苯和乙烯的光 催化转化依然不受影响, 结果见图 6. 显然, 光催化 反应效率并不随磁场强度的增强而改变.

\section{3 讨 论}

综合上述结果表明, 在低于 $0.2 \mathrm{~T}$ 的磁场下, 磁 场对气相光催化反应均没有本征促进作用. 至于在 更高场强下是否存在磁场效应有待进一步研究. $\mathrm{Klwl}{ }^{[8]}$ 研究了磁场对 $\mathrm{TiO}_{2}-\mathrm{RuO}_{2}-\mathrm{Pt}$ 催化剂上紫外光 光催化分解水的反应, 发现即使施加 $0.4 \mathrm{~T}$ 的磁场 也不导致 $\mathrm{H}_{2}$ 释放率有任何改变, 用 $\mathrm{CdS}-\mathrm{RuO}_{2}-\mathrm{Pt}$ 作 催化剂也得到同样的结果, 认为磁场不影响电子-空 穴的复合, 不影响电子从颗粒到负载催化剂的迁移 和催化剂表面附近所产生的顺磁性物种的配对反应 等过程. 但 $\mathrm{Klwl}$ 观察到, 当给 $\mathrm{TiO}_{2}-\mathrm{RuO}_{2}-\mathrm{Pt}$ 催化体 系中添加 $\mathrm{Ru}(\mathrm{bpy})_{3}^{2+}$ 和 EDTA, 或给 $\mathrm{CdS}-\mathrm{RuO}_{2}-\mathrm{Pt}$ 催 化体系中加人 $\mathrm{Ru}(\mathrm{bpy})_{3}^{2+} 、 \mathrm{EDTA}$ 和 $\mathrm{MV}^{2+}$ (dimethyl viologen)时, 在可见光照射下产氢量随磁场强度的 提高却会明显降低, 认为这与外加物质的强磁响应

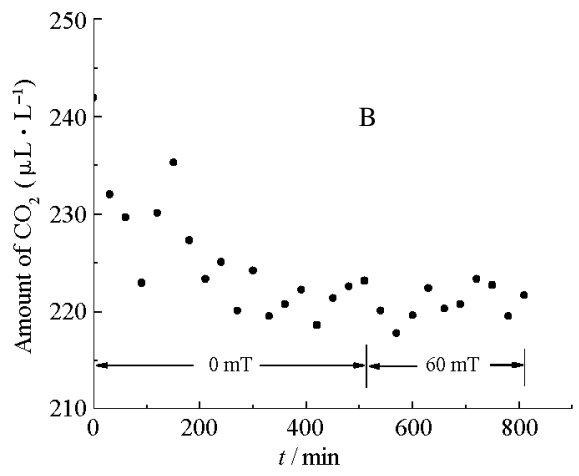

图 5 用反应装置 $(\mathrm{N})$ 所观测外加磁场对乙烯光催化转化率和 $\mathrm{CO}_{2}$ 含量影响

Fig.5 Effect of magnetic field on the photocatalytic conversion of ethylene and $\mathrm{CO}_{2}$ yield over $\mathrm{Pt} / \mathrm{TiO}_{2}$ with the apparatus (N)

A) ethylene conversion; $\mathrm{B}) \mathrm{CO}_{2}$ yield 

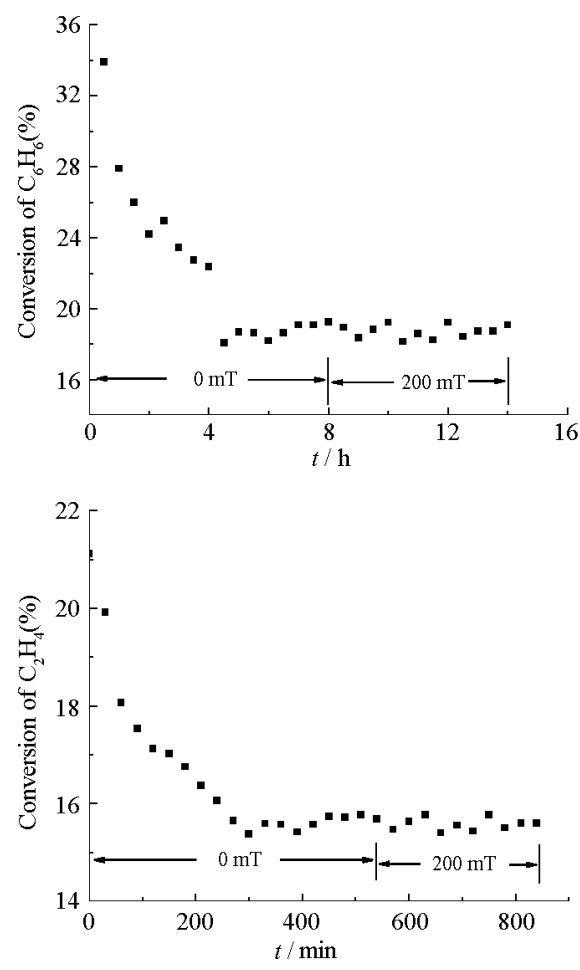
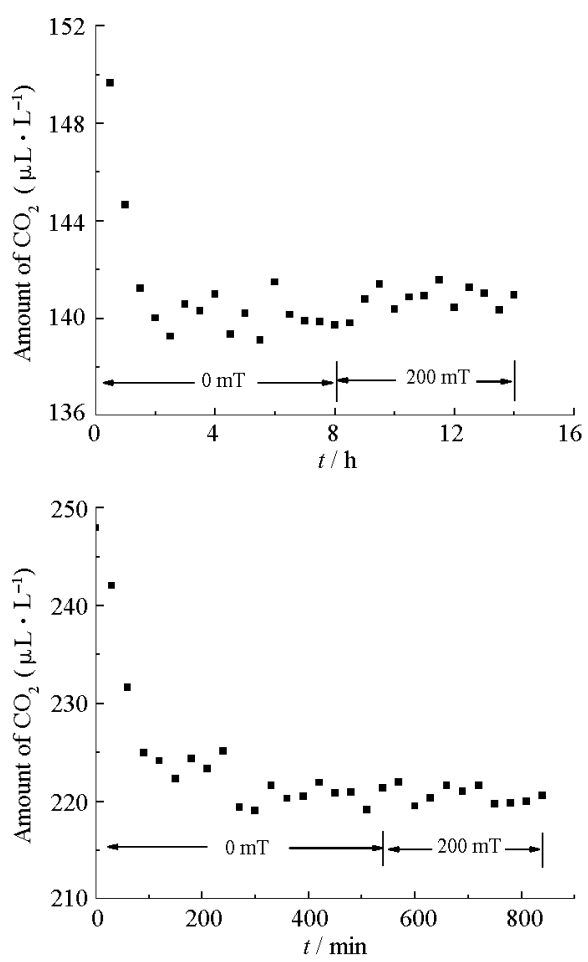

图 6 用反应装置(N)所观测 $0.2 \mathrm{~T}$ 强度磁场对苯和乙烯光催化反应的影响

Fig.6 Effect of magnetic field with $0.2 \mathrm{~T}$ intensity on the photocatalytic reactions of benzene and ethylene over $\mathrm{Pt} / \mathrm{TiO}_{2}$ by the apparatus $(\mathrm{N})$

C) benzene; D) ethylene

有关. Wakasa 等 ${ }^{[5]}$ 根据自由基对理论和他们的实验 观察认为, 磁场既不是由于超精细耦合 (HFCM) 作用 也不是通过 $\Delta \mathrm{g}$ 机理 $(\Delta \mathrm{gM})$ 影响叔丁醇的光催化反 应, 高强度磁场对反应的加速作用可能由于磁场诱 导阻碍了半导体光生电子和空穴的复合. 上述两个 反应虽然不同, 但都是固-液光催化体系, 两个作者 所得到的结果和对结果的解释却有明显的不同, 说 明多相光催化过程磁场效应的复杂性.

通过外加磁场来提高光催化反应效率设想是基 于光催化反应与光化学反应的相似性, 即它们都是 自由基参与的反应, 磁场的存在可能会改变具有顺 磁性自由基的状态和分布. 大量研究报道己经证明 磁场效应在光化学反应中是存在的, 不过都得自于 胶束体系和溶液体系. 磁场的影响取决于自由基对 与磁场的相互作用, 只有当自由基物种的自旋弛豫 比较慢, 外加磁场才有可能使它的自旋弛豫速度依 赖于磁场 ${ }^{[8]}$. 对气固光催化反应, 有可能光催化过程 的决速步为光诱导电子和空穴的分离和捕获, 一旦 产生差基自由基或超氧自由基, 次级反应会很快进 行; 也有可能差基自由基或超氧自由基的自旋驰豫 太快, 次级有机自由基又转化很快, 因而磁场不会对
反应产生明显影响.

最后, 有必要对用反应装置(M)所得结果作一些 说明. 对磁场影响灯管的发光强度物理学已有明确 的解释 ${ }^{[9-10]}$. 灯丝发射电子使气体弧光放电进而激发 湬蒸气和管壁的苂光粉产生紫外线，外加磁场由于 会对灯管内运动的电子和离子产生罗仑兹力, 依赖 于磁场强度而导致发光强度增大、减弱甚至熄灭. 而 灯管的发射波长则决定于苂光粉的成分和录蒸气本 身的性质 ${ }^{[11]}$, 不会受磁场的影响, 这一点也由实验测 定所证实(图略). 实验测定表明, 在我们所研究磁场 强度范围, 灯管的发光强度随磁场增大而增强. 这 样, 在其它实验条件不变的情况下, 施加磁场比不施 加磁场时光催化剂床层会获得更强的光照. 由于反 应所需要活化能能量来自光能, 有人在研究光催化 降解三氯甲烷的反应时证实降解速率与光强的平方 根成线性关系 ${ }^{[2]}$. 此外, 光强的增加会导致灯管温度 升高, 进而引起反应器温度升高. 实验检测表明, 与 不加磁场比较, 施加 $60 \mathrm{mT}$ 的磁场时反应器的温度 升高近 $17{ }^{\circ} \mathrm{C}$. 早先的研究表明, 与 $\mathrm{TiO}_{2}$ 相比, Pt/ $\mathrm{TiO}_{2}$ 催化剂上的光催化反应有十分明显的温度效 应, 即升高温度光催化效率有显著提高, 其主要与 
$\mathrm{Pt} / \mathrm{TiO}_{2}$ 热催化性能有关 ${ }^{[6]}$. 所以, 图 2 和图 3 结果可 能与两种因素都有关. 关于苯磁场-光催化过程 (图 2)中第二次施加磁场时比第一次施加磁场 $\mathrm{CO}_{2}$ 的浓 度增加幅度更大, 其可从温度升高来理解. 因为随反 应时间延长, 伴随着催化剂的失活, 催化剂表面的结 碳增多, 当第二次施加磁场时由于光源发光强度增 加而引起的反应温度升高加速了表面结碳的热催化 氧化, 因而释放出更多的 $\mathrm{CO}_{2}$.

\section{3 结 论}

当紫外灯管靠近磁场时, 外加磁场对 $365 \mathrm{~nm}$ 紫外光照射下 $\mathrm{Pt} / \mathrm{TiO}_{2}$ 光催化降解空气中苯和乙烯 有显著的促进作用, 但这种作用主要是磁场增强了 紫外灯管的发光强度, 而发光强度的提高导致了反 应温度的升高所致. 当排除磁场对光源的影响后, 磁 场本身不会引起反应温度升高, 也对光催化反应不 产生本征影响, 因而在低于 $0.2 \mathrm{~T}$ 的磁场强度下, 磁 场对苯和乙烯的气相光催化过程没有促进作用.

\section{References}

1 Vinodgopal, K.; Hotchandani, S.; Khmat, P. V. J. Phys. Chem., 1993, 97: 9040

2 Li, D. Z.; Fu, X. Z.; Zheng, Y. Chemical Journal of Chinese Universites, 2002, 23(12): 323 [李旦振, 付贤智, 郑 宜. 高等
学校化学学报(Gaodeng Хиехiаo Ниахие Хиеbao), 2002, 23(12): $323]$

3 Wang, H. H.; Yu, J. Y.; Luo, Z. Y. Photograph. Sci. \& Photochem., 1998, 16(2): 18 [王涵慧, 俞稼镛, 罗曾义. 感光科学与光化学 (Ganguang Кехие Yи Guanghuaxиe), 1998, 16(2): 182]

4 Zhang, W.; Wang, X. X.; Fu, X. Z. Chem. Commun., 2003:2196

5 Wakasa, M.; Suda, S.; Hayashi, H.; Ishii, N.; Okano, M. J. Phys. Chem. B, 2004, 108: 11882

6 Fu, X.; Clark, L. A.; Zeltner, W. A.; Anderson, M. A. J. Photochem. Photobio. A: Chem., 1996, 97: 181

7 Sato, S. J. Catal., 1985, 92: 11

8 Klwl, J. J. Phys. Chem., 1983, 87: 2274

9 Fan, Y. P.; Lan, H. J. J. Liuzhou Teachers College, 2004, 19: 113 [樊雅平, 蓝海江. 柳州师专学报 (Liuzhou Shizhuan Xuebao), 2004, 19: 113]

10 Liu, C.; Zou, J.Y.; Liu, Z. L. J. Huazhong Univ. of Sci. \& Tech., 1999, 27: 81 [刘 春, 邹积岩, 刘祖黎. 华中理工大学学报 (Huazhong Ligong Daxue Xиebao), 1999, 27: 81]

$11 \mathrm{Yi}, \mathrm{W}$. B. Basic knowledge of electronic ballast for daylight lamp. Tianjin: Tianjin People Press, 1974:4 [弯文波. 日光灯镇流器基 础知识. 天津: 天津人民出版社, 1974:4]

12 Bahanemann, D.; Bockelamann, D.; Goslich, R. Solar Ener. Mater. 1991, 24: 564 